\title{
Correlation between the X-ray Spectra of Young Pulsars and their Wind Nebulae
}

\author{
E. V. Gotthelf \\ Columbia Astrophysics Laboratory, Columbia University, 550 West \\ $120^{\text {th }}$ Street, New York, NY 10027, USA
}

\begin{abstract}
A significant correlation is discovered between the X-ray spectra of young pulsars (PSRs) and that of their associated wind nebulae (PWNe). For a sample of nine bright Crab-like pulsar systems observed with the Chandra X-ray observatory, we report a linear relationship between the photon indices for the PWNe and those of the phase-averaged pulsar emission, where $\Gamma_{P W N}=0.72 \pm 0.13 \times \Gamma_{P S R}+0.86 \pm 0.20$. Furthermore, we find that the spectral slopes of younger, more energetic, pulsars tend to be steeper. These results confirm a consistent pulsar emission mechanism and provide important new observational constraints on the current theory of shock acceleration models for pulsar wind emission.
\end{abstract}

\section{Introduction}

New observations of young pulsars in the X-ray band are providing fresh insight into their local environment. Nearly all show evidence of a surrounding pulsar wind nebulae (PWNe). Most notably, early Chandra observations of the Crab pulsar (Weisskopf et al. 2000) revealed a complex emission structure, involving co-aligned toroidal arcs, axial jets, and wisps, similar to that seen from the optical Crab nebula (Hester 1998). Subsequent Chandra observations of the Vela pulsar resulted in the discovery of strikingly similar features, including the tori, jets, and wisps (Helfand et al. 2001). The basic morphology described above, referred to herein as the pulsar wind nebulae, is now found to be common to young, energetic, Chandra pulsars associated with supernova remnants (Gotthelf 2001).

Here we present a spectral study of nine pulsars observed by Chandra which show bright, resolved PWNe (see Table 1). These pulsars were imaged using the Advanced CCD Imaging Spectrometer (ACIS) whose arc-sec resolution allows us to isolate the pulsar emission from that of the nebula. ACIS is sensitive to $\mathrm{X}$-rays in the $0.2-10 \mathrm{keV}$ energy band with an energy resolution of $\Delta E / E \sim 0.1$ at $1 \mathrm{keV}$. For each object, we extracted spectra from the pulsar, PWN, and their local backgrounds, when available, or obtained spectral parameters from the literature as indicated. The extraction regions for the pulsar spectra were based on data above $4.0 \mathrm{keV}$, where the count rate per pixel from the pulsar dominates over the surrounding diffuse nebula emission. The nebula regions were defined by the $3 \sigma$ contour above the background level with the pulsar region excluded. Data were corrected for CTI effects (Townsley et al. 2001) and analyzed using standard tools and procedures (CIAO 2.2/CALDB v.2.9). All 
spectra were grouped to a minimum of 50 counts per spectral bin and fitted with an absorbed power-law model whose flux is given by $F(E) \propto e^{\sigma(E) n_{H}} E^{-\Gamma}$, were $\Gamma$ is the photon index and $n_{H}$ is the hydrogen column density to the source.

Table 1. X-ray Spectra of Young Pulsars Observed with Chandra ${ }^{a}$

\begin{tabular}{lccccc}
\hline \hline Remnant & $\Gamma_{\text {PWN }}^{b}$ & $\Gamma_{\text {PSR }}^{c}$ & $\Gamma_{\text {Pulsed }}^{d}$ & $\tau(\mathrm{kyr})^{e}$ & $E\left(10^{35} \mathrm{erg} / \mathrm{s}\right)$ \\
\hline G11.2-0.3 & $1.28 \pm 0.15$ & $0.63 \pm 0.12$ & $0.60 \pm 0.60$ & 23.0 & 53 \\
Vela XYZ & $1.50 \pm 0.04$ & $0.95 \pm 0.24$ & $0.93 \pm 0.26$ & 12.0 & 67 \\
G54.1+0.3 & $1.64 \pm 0.18$ & $1.09 \pm 0.09$ & $1.06 \pm 0.86$ & 2.9 & 118 \\
Kes 75 & $1.92 \pm 0.04$ & $1.39 \pm 0.11$ & $1.10 \pm 0.30$ & 0.7 & 82 \\
MSH 15-52 & $1.93 \pm 0.03$ & $1.40 \pm 0.50$ & $1.26 \pm 0.08$ & 1.6 & 140 \\
3C 58 & $1.92 \pm 0.11$ & $1.73 \pm 0.15$ & $1.11 \pm 0.34$ & 5.0 & 263 \\
SNR 0540-69 & $2.09 \pm 0.11$ & $1.88 \pm 0.11$ & $1.83 \pm 0.13$ & 1.7 & 1481 \\
Crab Nebula & $2.14 \pm 0.01$ & $1.85 \pm 0.01$ & $1.87 \pm 0.01$ & 1.3 & 4394 \\
N157B Neb. & $2.28 \pm 0.12$ & $2.07 \pm 0.21$ & $1.60 \pm 0.35$ & 5.0 & 4916 \\
\hline \hline
\end{tabular}

${ }^{a}$ Ranked by increasing pulsar photon index $\left(\Gamma_{\mathrm{PSR}}\right)$. Data for the Crab pulsar and nebula were obtained from Pravdo, Angelini \& Harding (1997)

${ }^{b}$ Values for the following objects were taken from the literature: G54.1+0.3: Lu et al. 2002; SNR 0540-69: Karret et al. (2001).

${ }^{c}$ Includes both pulsed and un-pulsed emission, corrected for pile-up.

${ }^{d}$ Pulsar's pulsed spectrum references: Vela: Strickman, Harding \& Jager (1999); G11.2-0.3: Torii et al. (1997); Kes 75: Gotthelf et al. (2000); N157B: Marshall et al. (1998); SNR 0540-69: Karret et al. (2001)

${ }^{e}$ Characteristic pulsar spin-down age, $\tau \equiv P / 2 \dot{P}$.

The fitting procedure was as follows. In order to avoid potential soft thermal emission contamination, the spectral fits for both the pulsars and PWNe were restricted to energies above $2 \mathrm{keV}$, where the fits are largely insensitive to the hydrogen column density, $\mathrm{n}_{H}$. In these fits, the $\mathrm{n}_{H}$ was held fixed to the value determined from fits to the nebula spectra over the full ACIS band. The spectral fits to the pulsar emission included an additional model to take into account the effects of CCD pile-up. Table 1 lists the resultant spectral slopes for each object, including the spectra slope of the pulsed emission only, taken from published values, when available, or derived by phase-resolved spectroscopy using $A S C A$ or $R X T E$ data.

\section{Results}

A plot of the 2-10 keV spectral slope (photon index $\Gamma$ ) for each pulsar versus that of its PWN is shown in the left panel of Figure 1. These range from $0.6<$ $\Gamma_{P S R}<2.1$ and $1.3<\Gamma_{P W N}<2.3$, for the pulsars and PWNe, respectively. A linear regression fit to these data points, taking into account the uncertainties in both coordinates, yields,

$$
\Gamma_{P W N}=0.72 \pm 0.13 \times \Gamma_{P S R}+0.86 \pm 0.20
$$

With a linear correlation coefficient of $r=0.96$, this relationship is highly significant and covers the range of known pulsar spectral power-law indexes in 

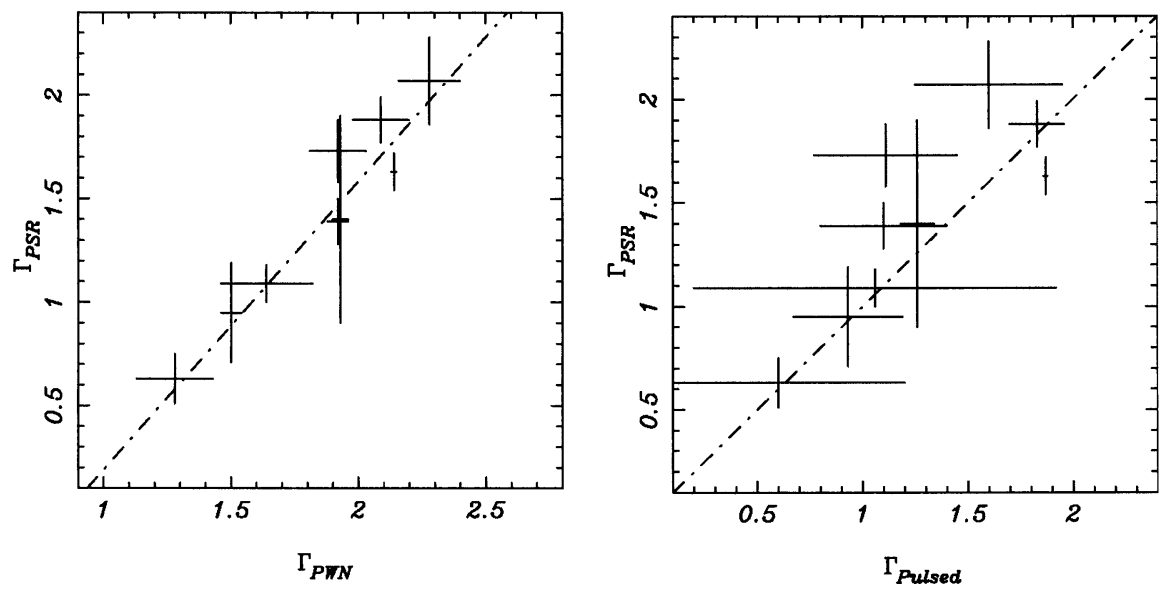

Figure 1. LEFT - Relationship between a pulsar's spectral slope $\left(\Gamma_{\mathrm{PSR}}\right)$ and that of its wind nebulae $\left(\Gamma_{\mathrm{PWN}}\right)$, assuming a simple powerlaw model for the objects presented in Table 1 . The dashed-line indicates the best-fit. The physical origin of this relationship has yet to be determined. RIGHT - A comparison between the spectral slope of the pulsed emission $\left(\Gamma_{\text {Pulsed }}\right)$ and that of the total pulsar emission $\left(\Gamma_{\mathrm{PSR}}\right)$ for the same objects. A one-to-one correspondence is indicated by the dashed line.

the $2-10 \mathrm{keV}$ X-ray band. The results of Table 1 are rank-ordered by PSR photon indices which is nearly equivalent to the ordering by spin-down energy, also given in the table. To a lesser extent, the spin-down age roughly follows the spin-down energy. This suggests that the more energetic, generally younger pulsars have systematically steeper spectra.

On the right panel of Figure 1, the spectral slope for each pulsar is now plotted against the spectral slope of the pulsed emission only. This is a good test for systematic spectral distortion resulting from pile-up, as the pulsed measurements, based on phase-resolved spectroscopy, are immune to pile-up. Although the spectra of the pulsed and total emission need not be the same, it is reassuring that they generally agree to within measurement errors. This is consistent with a simple rotating NS model for which a single emission mechanism is modulated over the surface of the neutron star. Interesting exceptions are the Crab and 3 C58 pulsars. The former is known to have a phase dependence in the measured photon index; we predict that the same is true for the pulsar in 3C58 and any other object whose pulsed emission deviates significantly from its phase-averaged emission in this band.

Our results are rather surprising, since no correlation is expected between the spectrum of a pulsar and that of its PWN or between a pulsar's spectral shape and its spin-down energy. Both the polar-cap (e.g., Harding et al. 1978) and outer-gap (e.g., Cheng, Ho, \& Ruderman 1986) models requires a population of accelerated electrons, radiating synchrotron emission from close to the star (within the light cylinder), whose spectrum depends on the local input particle 
distribution and magnetic geometry. In both theories, these particles are the origin of the wind which energizes the nebula. Although the nebular emission arises from the shocked wind, it is not expected to retain sufficient information on the original input particle distribution to track the pulsar's spectrum. These theories, however, are recognized to be far from complete, and furthermore, are inadequate in explaining the observed localized tori and jet-like features.

The spectral connection presented herein suggests that the emission mechanisms of a pulsar and its nebula are linked either directly or indirectly, via a common mediating process. This coupling may imply that the structured nebula is being powered directly by the pulsar. Furthermore, the apparent steepening of the spectral slope with spin-down energy $(\dot{E})$ suggests that the spectral evolution is best parameterized by $\dot{E}$ instead of the characteristic age $\tau(\equiv P / 2 \dot{P})$. There is ample evidence that $\tau$ can be quite uncertain as a measure of the true age of the pulsar (e.g. Kaspi et al. 2001).

In summary, this work reveals a fundamental spectral relationship between pulsars and their wind nebulae not easily explained in the framework of current models of pulsar emission mechanisms. The spectral slopes are found to be tightly correlated over the full range of measured values and the spectral index of the younger, more energetic pulsars are seen to be steeper. The former strongly constrains emission models and the latter provides an important probe of pulsar spin-down evolution. These results provide new observational insight into the emission mechanisms of young rotation-powered neutron stars. As more young rotation-powered pulsars are observed with Chandra, a more complete picture can emerge of the morphological and spectral characteristics of young pulsar evolution, paving the way for future models and theory.

Acknowledgments. This work made possible by NASA LTSA grant NAG 57935. We thank Charles M. Olbert for assistance with the spectral analysis.

\section{References}

Cheng, K. S., Ho, C., \& Ruderman, M. 1986, ApJ, 300, 500

Harding, A. K., Tademaru, E., \& Esposito, L. S. 1978, ApJ, 225, 226

Helfand, D. J., Gotthelf, E. V., \& Halpern, J. P. 2001, ApJ, 556, 380

Hester, J. J. 1998, MmSAI, 69, 883

Gotthelf, E. V., et. al. 2000, ApJ, 542, L37

Gotthelf, E. V. 2001, AIP Conf. Proc., Vol. 586, p. 513

Kaaret, P., et. al 2001, ApJ, 546, 1159

Kaspi, V. M., et al. 2001, ApJ, 560, 371

Lu, F. J., et al. 2002 ApJ, 568, 49

Marshall, F. E., et al. 1998, ApJ, 499, L179

Pravdo, S. H., Angelini, L., \& Harding, A. K. 1997, ApJ, 491, 808

Strickman, M. S., Harding, A. K., \& de Jager, O. C. 1999, ApJ, 524, 373

Townsley, L. K., Broos, P. S, Garmire, G. P., Nousek, J. A., ApJ, 534, L139

Torii, K., Tsunemi, H., Dotani, T.,\& Mitsuda, K. 1997 ApJ, 489, L145

Weisskopf, M. C. et. al 2000, ApJ, 536, L81. 